\title{
Measuring the Tensor Polarization of Positronium
}

\author{
Alexander J. Silenko* \\ Institute of Nuclear Problems, Belarusian State University, Minsk 220030, Belarus
}

(Dated: November 4, 2018)

\begin{abstract}
A method for measuring the tensor polarization in a positronium (Ps) beam is proposed, which is based on the determination of the Ps lifetime as a function of the orientation of a homogeneous magnetic field. The dependence of the orthopositronum (o-Ps) lifetime on the angle between the directions of the magnetic field and the tensor polarization of the Ps beam can be determined from the results of measurements from two or more field orientations.
\end{abstract}

PACS numbers: $36.10 . \mathrm{Dr}$

*Electronic address: silenko@inp.minsk.by 
A positronium (Ps) atom, which is composed of an electron and a positron, possesses a number of unique physical and chemical properties. This stimulates extensive theoretical and experimental investigations of the characteristics of Ps and makes possible its successful use in applied research $[1,2,3,4,5]$. Ps atoms can exist in para (p-Ps) and ortho (oPs) states, which are characterized by spins of 0 and 1, respectively. The lifetimes of p-Ps and o-Ps are significantly different: in vacuum, these values are $1,25 \cdot 10^{-10}$ and 1,42 . $10^{-7} \mathrm{~s}$, respectively. Since electrons and positrons represent a coupling of particle and antiparticle, all the multipole moments of Ps have zero values. Nevertheless, o-Ps atoms can possess vector and tensor polarizations. The tensor polarization is among the most important characteristics of a beam of Ps atoms. It is the tensor (rather than the vector) polarization that appears in the presence of a magnetic field. This is caused by a decrease in the lifetime of o-Ps with zero spin projection onto the direction of the magnetic field as a result of its mixing with the p-Ps state $[1,6]$.

This Letter describes a simple and quite readily implemented method for determining the tensor polarization of Ps beams, which is based on the dependence of the mean Ps lifetime on the directions of a homogeneous magnetic field. Below, the atomic system of units $\hbar=c=1$ is used throughout the text. The polarization of particles (atoms, nuclei) is described in terms of a three-component polarization vector $\boldsymbol{P}$ and a polarization tensor $P_{i j}$, which has five independent components:

$$
P_{i}=\frac{<S_{i}>}{S}, \quad P_{i j}=\frac{3<S_{i} S_{j}+S_{j} S_{i}>-2 S(S+1) \delta_{i j}}{2 S(2 S-1)}, \quad i, j=x, y, z,
$$

where $P_{i j}=P_{j i}$ and $P_{x x}+P_{y y}+P_{z z}=1$.

If the direction of spin quantization in a medium is fixed (e.g., by a magnetic field directed along the $z$ axis), the spin wave function of a particle can be represented as the superposition of the spin wave functions of the basis set corresponding to the spin projections $S_{z}=-1,0$ and +1 . A particle entering a medium may possess a different polarization. We are interested in the case where a particle has a fixed spin projection $\left(S_{l}=-1,0\right.$ or +1$)$ onto a certain direction $\boldsymbol{l}$ characterized by spherical angles $\theta$ and $\phi$. The eigenfunctions of the states with fixed spin projections on $\boldsymbol{l}$ are determined by the equation

$$
S_{l} \Psi_{\lambda}=\lambda \Psi_{\lambda}
$$

where $\lambda=-1,0$ or +1 , and

$$
S_{l}=S_{x} \sin \theta \cos \phi+S_{y} \sin \theta \sin \phi+S_{z} \cos \theta .
$$


The solutions to Eqs. (2) and (3) have the form of wavefunctions [7, 8]:

$$
\begin{gathered}
\psi_{-1}=e^{i \alpha_{1}}\left(\begin{array}{c}
-\sin ^{2}(\theta / 2) e^{-i \phi} \\
\sqrt{2} \sin (\theta / 2) \cos (\theta / 2) \\
-\cos ^{2}(\theta / 2) e^{i \phi}
\end{array}\right), \quad \psi_{0}=\frac{1}{\sqrt{2}} e^{i \alpha_{2}}\left(\begin{array}{c}
-\sin \theta e^{-i \phi} \\
\sqrt{2} \cos \theta \\
\sin \theta e^{i \phi}
\end{array}\right), \\
\psi_{1}=e^{i \alpha_{3}}\left(\begin{array}{c}
\cos ^{2}(\theta / 2) e^{-i \phi} \\
\sqrt{2} \sin (\theta / 2) \cos (\theta / 2) \\
\sin ^{2}(\theta / 2) e^{i \phi}
\end{array}\right),
\end{gathered}
$$

where $\alpha_{1}, \alpha_{2}$, and $\alpha_{3}$ are arbitrary angles. When a particle polarized along direction $\boldsymbol{l}$ falls into a medium with a different polarization, the components of the particle wave function describe the amplitudes of the probability of finding the corresponding spin projection onto a specific direction in the medium. The probability of finding a particle with the spin projection $m$ on $\boldsymbol{l}$ in the state with spin projection $\lambda$ onto the $z$ is

$$
w_{\lambda}^{(m)}=\left|\psi_{m}(\lambda)\right|^{2}, \quad m=-1,0,1
$$

where the wave function components run upward though values of $\lambda=-1,0$, and +1 .

For an unpolarized beam, the numbers of particles with any spin projection are the same. In a partly polarized beam, which can be considered as consisting of a coherent (polarized) and incoherent (unpolarized) components, the coherent component is described by formulas (4) and (5), while the incoherent component possesses neither vector nor tensor polarizations.

In the general case, a beam polarized along direction $\boldsymbol{l}$, is characterized, instead of a fixed $m$ set, by three probabilities $\left(n_{+}^{\prime}, n_{0}^{\prime}, n_{-}^{\prime}\right)$ of detecting a separate particle in the state with the corresponding spin projection onto this direction. In particular, for an unpolarized beam $n_{+}^{\prime}=n_{0}^{\prime}=n_{-}^{\prime}=1 / 3$. According to formulas (4) and (5), the probabilities of finding a particle in the state with certain spin projection onto the $\mathrm{z}$ axis of the laboratory frame can be written as

$$
\begin{aligned}
& n_{+}=n_{+}^{\prime} \cos ^{4}(\theta / 2)+ \frac{1}{2} n_{0}^{\prime} \sin ^{2} \theta+n_{-}^{\prime} \sin ^{4}(\theta / 2), \quad n_{0}=\frac{1}{2} n_{+}^{\prime} \sin ^{2} \theta+n_{0}^{\prime} \cos ^{2} \theta+\frac{1}{2} n_{-}^{\prime} \sin ^{2} \theta, \\
& n_{+}=n_{+}^{\prime} \sin ^{4}(\theta / 2)+\frac{1}{2} n_{0}^{\prime} \sin ^{2} \theta+n_{-}^{\prime} \cos ^{4}(\theta / 2) .
\end{aligned}
$$

Thus, a change of the direction of spin projection quantization leads to a radical change in the polarization of particles. In the absence of p-Ps atoms at the initial moment (this very situation takes place in most experiments), the state of a beam can be described by 
setting only two components of the vector $P_{z}$ and tensor $P_{z z}$ polarization:

$$
P_{z}=n_{+}-n_{-}, \quad P_{z z}=3\left(n_{+}+n_{-}\right)-2=1-3 n_{0}, \quad n_{+}+n_{0}+n_{-}=1,
$$

where $n_{+}, n_{0}, n_{-}$are the fractions of o-Ps atoms in the states with the corresponding projections onto the z axis. According to formulas (6) and (7), the vector and tensor polarizations in the laboratory frame are determined by the equations

$$
P_{z}=P_{z}^{\prime} \cos \theta, \quad P_{z z}=\frac{1}{2} P_{z z}^{\prime}\left(3 \cos ^{2} \theta-1\right),
$$

where

$$
P_{z}^{\prime}=n_{+}^{\prime}-n_{-}^{\prime}, \quad P_{z z}^{\prime}=3\left(n_{+}^{\prime}+n_{-}^{\prime}\right)-2=1-3 n_{0}^{\prime} .
$$

The above relations are valid for the particles (atoms, nuclei) both in vacuum and in isotropic media. A specific feature of Ps is the possibility of completely describing the dynamics of annihilation using only two polarization parameters, $P_{z}$ and $P_{z z}$.

A change in the magnetic field orientation leads to a change in the angle $\theta$ between direction $\boldsymbol{l}$ (i.e., the o-Ps polarization direction) and the $z$ axis. Let us denote by $\tau_{ \pm}$the lifetime of components with nonzero spin projections onto the magnetic field direction $\left(S_{z}=\right.$ \pm 1 , respectively). These values are independent of the magnetic induction. Let $\tau_{0}(B)$ be the lifetime of the component with a zero spin projection $\left(S_{z}=0\right)$ onto the field direction. It is evidently that $\tau_{0}(0)=\tau_{ \pm}$, and both this value and $\tau_{0}(B)$ are independent of the angle $\theta$.

For $\theta=0$, the lifetime of a polarized Ps beam is

$$
T(B, 0)=\left(1-n_{0}^{\prime}\right) \tau_{ \pm}+n_{0}^{\prime} \tau_{0}(B)=\frac{2}{3} \tau_{ \pm}+\frac{1}{3} \tau_{0}(B)+\frac{1}{3} P_{z z}^{\prime}\left[\tau_{ \pm}-\tau_{0}(B)\right]
$$

According to formulas (6)-(9), the dependence of the beam lifetime on $\theta$ is described by the formula

$$
T(B, \theta)=\left(1-n_{0}\right) \tau_{ \pm}+n_{0} \tau_{0}(B)=\frac{1}{3}\left\{2 \tau_{ \pm}+\tau_{0}(B)+\frac{1}{2} P_{z z}^{\prime}\left(3 \cos ^{2} \theta-1\right)\left[\tau_{ \pm}-\tau_{0}(B)\right]\right\} .
$$

For the natural choice of $\theta=\pi / 2$, this expression simplifies to

$$
T(B, 0)-T\left(B, \frac{\pi}{2}\right)=\frac{1}{2} P_{z z}^{\prime}\left[\tau_{ \pm}-\tau_{0}(B)\right] .
$$

As was noted above, the tensor polarization is the main characteristic of a Ps beam, since the vector polarization does not influence the dynamics of Ps annihilation in a magnetic field. 
Formulas (10) and (12) determine a relationship between the tensor polarization of Ps and the Ps lifetime in the two most important particular cases: with a fixed direction and a fixed magnetic field. The more general dependence corresponding to the case when reorientation of the magnetic field is accompanied by a change in the field magnitude (from $B^{\prime}$ to $B$ ) is as follows:

$$
T(B, \theta)=T\left(B^{\prime}, 0\right)+\frac{1}{3}\left(1-P_{z z}^{\prime}\right)\left[\tau_{0}(B)-\tau_{0}\left(B^{\prime}\right)\right]-\frac{1}{2} P_{z z}^{\prime}\left[\tau_{ \pm}-\tau_{0}(B)\right] \sin ^{2} \theta
$$

This formula can be necessary in high-precision measurements (when even small changes in the magnetic induction upon rotation of the field have to be taken into account) or in a system with static magnets (when a perpendicular magnet with identical parameters is switched).

A question naturally arises as to whether the magnetic field rotation is necessary for highprecision measurements of the tensor polarization of Ps. In order to elucidate this point, let us rewrite formula (10) as

$$
P_{z z}^{\prime}=\frac{3 T(B, 0)-2 \tau_{ \pm}-\tau_{0}(B)}{\tau_{ \pm}-\tau_{0}(B)},
$$

and reduce Eq. (12) using this formula to

$$
P_{z z}^{\prime}=\frac{2\left[T(B, 0)-T\left(B, \frac{\pi}{2}\right)\right]}{3 \tau_{ \pm}-T(B, 0)-2 T\left(B, \frac{\pi}{2}\right)} .
$$

In the general case of arbitrary $\theta$, the equation for the tensor polarization of Ps takes the following form:

$$
P_{z z}^{\prime}=\frac{2[T(B, 0)-T(B, \theta)]}{3 \tau_{ \pm} \sin ^{2} \theta+\left(3 \cos ^{2} \theta-1\right) T(B, 0)-2 T(B, \theta)}
$$

The lifetime $\tau_{0}(B)$ can be determined with suffi- ciently high accuracy, either by using a second, unpolarized beam of Ps, or by carrying out measurements under ultrahigh vacuum conditions. In the absence of magnetic field, $\tau_{ \pm}$represents the measured o-Ps lifetime (in a medium), and in the field, this value represents the o-Ps lifetime with spin projections \pm 1 on the field direction. Therefore, $\tau_{0}(B)$ can be calculated proceeding from the average o-Ps lifetime in the field $B$, assuming that the initial beam has a one-third fraction of the component with $S_{z}=0$. If only an o-Ps beam is available whose tensor polarization is to be determined, the $\tau_{0}(B)$ value can be estimated with sufficient accuracy by calculations alone using the well-known formulas for Ps lifetimes in a magnetic field (see, e.g., [1, 6]). However, 
these formulas can be used only provided that measurements are performed under ultrahigh vacuum conditions. These circumstances substantially restrict the applicability of formula (14) to the determination of $P_{z z}$.

Using Eqs. (15) and (16), it is possible to bypass the aforementioned difficulties. Indeed, the right-hand parts of these equations contain only three quantities that are directly measured for a single tensor-polarized Ps beam, which can also possess vector polarization. In order to determine these quantities, three independent measurements are sufficient. The o-Ps lifetime in a medium, which is measured with a field switched on, is independent of tensor polarization. The two other lifetimes are determined for the fields oriented parallel and perpendicularly to the direction of beam polarization (or at a definite angle $\theta$ relative to this direction). This circumstance makes possible the tensor polarization of Ps to be measured with high accuracy. It should be emphasized that the proposed method does not require special vacuum conditions.

The measurements of tensor polarization are especially important for the investigations of Ps in substances containing paramagnetic atoms, in anisotropic media (including those with nanostructures), and in the study of Ps polarization dynamics. The tensor polarization also depends on the presence of magnetic field in the region of o-Ps formation and the initial polarization of the Ps beam. However, no investigations of the tensor polarization have been performed up to now. The method proposed in this paper for determining this important characteristic provides the possibility of such investigations with a sufficiently high accuracy, which will contribute to detailed characterization of the interaction of Ps with various substances.

[1] V. I. Gol'danskii, Physical Chemistry of the Positron and Positronium (Nauka, Moscow, 1968) [in Russian].

[2] Schrader D.M., Jean Y.C. Positron and Positronium Chemistry (Studies in Physical and Theoretical Chemistry, No. 57). New York: Elsevier, 1988. 395 p.

[3] Jean Y.C., Mallon P.E., and Schrader D.M. Principles and Applications of Positron and Positronium Chemistry. London: World Scientific Publ. Ltd., 2003. 424 p. 
[4] Garcia A.A. Positrons and Positronium for Polymer Thin Film Analysis. Delft: Delft Univ. Press, 2003. 160 p.

[5] Baryshevsky V.G. // Phys. Stat. Sol. (b). 1984. V. 124. N 2. P. 619-623.

[6] Bisi A., Fiorentini A., Gatti E., and Zappa L. // Phys. Rev. 1962. V. 128. N 5. P. 2195-2199.

[7] A. Ya. Silenko, Poverkhnost, No. 4, 45 (2005).

[8] A. Ya. Silenko, Poverkhnost, No. 4, 52 (2005). 